\title{
Generalized Sturmian Functions in prolate spheroidal coordinates
}

\author{
Darío Mitnik ${ }^{1,2}$, Andres Lopez ${ }^{2}$, and Ugo Ancarani ${ }^{3}$ \\ ${ }^{1} \mathrm{DF}, \mathrm{FCEyN}, \mathrm{UBA}$ \\ ${ }^{2} \mathrm{IAFE}$ \\ ${ }^{3}$ Universite de Lorraine, Metz, France
}

June 13,2020

\begin{abstract}
With the aim of describing bound and continuum states for diatomic molecules, we develop and implement a spectral method that makes use of Generalized Sturmian Functions (GSF) in prolate spheroidal coordinates. In order to master all computational issues, we apply here the method to one-electron molecular ions and compare it with benchmark data for both ground and excited states. We actually propose two different computational schemes to solve the two coupled differential equations. The first one is an iterative $1 \mathrm{~d}$ procedure in which one solves alternately the angular and the radial equations, the latter yielding the state energy. The second, named direct $\$ 2 \mathrm{~d} \$$ method, consists in representing the Hamiltonian matrix in a two-dimensional GSF basis set, and its further diagonalization. Both spectral schemes are timewise computationally efficient since the basis elements are such that no derivatives have to be calculated numerically. Moreover, very accurate results are obtained with minimal basis sets. This is related on one side to the use of the natural coordinate system and, on the other, to the intrinsic good property of all GSF basis elements that are constructed as to obey appropriate physical boundary conditions. The present implementation for bound states paves the way for the study of continuum states involved in ionization of one or two-electron diatomic targets.
\end{abstract}

\section{Hosted file}

MitnikProlates.pdf available at https://authorea.com/users/332269/articles/459277generalized-sturmian-functions-in-prolate-spheroidal-coordinates 